MATHEMATICS OF COMPUTATION

Volume 77, Number 264, October 2008, Pages 1871-1885

S 0025-5718(08)02126-1

Article electronically published on May 8, 2008

\title{
ON THE ORDER OF CONVERGENCE OF THE DISCONTINUOUS GALERKIN METHOD FOR HYPERBOLIC EQUATIONS
}

\author{
GERARD R. RICHTER
}

\begin{abstract}
The basic error estimate for the discontinuous Galerkin method for hyperbolic equations indicates an $O\left(h^{n+\frac{1}{2}}\right)$ convergence rate for $n$th degree polynomial approximation over a triangular mesh of size $h$. However, the optimal $O\left(h^{n+1}\right)$ rate is frequently seen in practice. Here we extend the class of meshes for which sharpness of the $O\left(h^{n+\frac{1}{2}}\right)$ estimate can be demonstrated, using as an example a problem with a "nonaligned" mesh in which all triangle sides are bounded away from the characteristic direction. The key to realizing $h^{n+\frac{1}{2}}$ convergence is a mesh which, to the extent possible, directs the error to lower frequency modes which are approximated, not damped, as $h \rightarrow 0$.
\end{abstract}

\section{INTRODUCTION}

The discontinuous Galerkin (DG) method for partial differential equations dates back to the work of Reed and Hill [5] and Lesaint and Raviart [3], who viewed it as a method for solving first order scalar hyperbolic equations over finite element meshes. The problem they considered was of the form:

$$
\begin{cases}\alpha \cdot \nabla u+\beta u=f, & \text { in } \Omega, \\ u=g, & \text { on } \Gamma_{\text {in }}(\Omega),\end{cases}
$$

where $\Omega$ is a 2-dimensional polygonal domain and $\alpha$ (a vector) and $\beta$ are constants. The inflow boundary of $\Omega, \Gamma_{\text {in }}(\Omega)$, is characterized by $\alpha \cdot \mathbf{n}<0$ where $\mathbf{n}$ denotes the unit outer normal (with $\Gamma_{\text {out }}(\Omega)$ characterized analogously).

Let $\left\{\tau_{h}\right\}$ be a quasiuniform family of triangulations of $\Omega$ with characteristic triangle diameter denoted by $h$. In each triangle $T \in \tau_{h}$, the corresponding $n t h$ degree DG approximation $u_{h} \in P_{n}(T)$ is defined by

(2) $\left(\alpha \cdot \nabla u_{h}+\beta u_{h}, v_{h}\right)_{T}-\int_{\Gamma_{\text {in }}(T)}\left(u_{h}^{+}-u_{h}^{-}\right) v_{h} \alpha \cdot \mathbf{n} d s=\left(f, v_{h}\right)_{T}, \quad v_{h} \in P_{n}(T)$.

Here $(,)_{T}$ denotes the $L^{2}(T)$ inner product, $s$ denotes arclength, and $u_{h}^{ \pm}(\mathbf{x})=$ $\lim _{\epsilon \rightarrow 0_{+}} u_{h}(\mathbf{x} \pm \epsilon \alpha)$. As initial data, we take $u_{h}^{-}$to be the $L^{2}$ projection of $g$ into an $n$ th-degree piecewise polynomial on $\Gamma_{\text {in }}(\Omega)$. The method is explicit, allowing $u_{h}$ to be computed triangle by triangle, or in parallel within layers of triangles, in accord with domain of dependence requirements.

Received by the editor February 15, 2007 and, in revised form, November 20, 2007.

2000 Mathematics Subject Classification. Primary 65M60, 65M15.

Key words and phrases. Finite element, hyperbolic.

(C)2008 American Mathematical Society Reverts to public domain 28 years from publication 
The basic error estimate for (2) is due to Johnson and Pitkäranta [2]:

$$
\left|u-u_{h}^{-}\right|_{\Gamma_{\text {out }}(\Omega)}+\left\|u-u_{h}\right\|_{\Omega} \leq C h^{n+1 / 2}\|u\|_{n+1, \Omega} .
$$

The norms in the above estimate are those on $L^{2}\left(\Gamma_{\text {out }}(\Omega)\right), L^{2}(\Omega)$, and $H^{n+1}(\Omega)$, respectively. Here and in what follows, $C$ indicates a generic positive constant, independent of $u$ and $h$.

The estimate (3) predicts an $O\left(h^{1 / 2}\right)$ gap from the optimal rate of convergence. In practice, however, the optimal $O\left(h^{n+1}\right)$ rate is frequently observed, even for computational meshes having no particular uniformity. See for example [6], in which computational results are given for random perturbations of uniform meshes.

In previous studies of this effect, the question of mesh alignment has played a prominent role. In general, $u_{h}$ propagates from triangle to triangle across edges that are not aligned with the characteristic direction. A characteristic mesh line cutting through $\Omega$ will divide it into subregions in which $u_{h}$ evolves independently, thus limiting crosswind effects. A proof of $O\left(h^{n+1}\right)$ convergence, requiring $u \in$ $H^{n+2}(\Omega)$, was given in [6] for an "almost uniform", nonaligned mesh with triangle sides bounded away from the characteristic direction. With these assumptions, the error generated in each layer of triangles is damped exponentially as $u_{h}$ evolves through subsequent layers, leading to the optimal rate. Recently, Cockburn, et al. [1] derived an optimal order estimate, requiring only $u \in H^{n+1}(\Omega)$, for a special mesh in $R^{d}$ consisting of simplices having one outflow face. For $d=2$, such a mesh can be constructed by arranging triangles along characteristic mesh lines with $O(h)$ spacing.

That (3) is indeed sharp, even for infinitely smooth exact solutions, was demonstrated computationally by Peterson 4 using piecewise linear approximation on a simple example problem. The triangulation employed in [4 was uniform, except for a set of characteristic mesh lines spaced at $O\left(h^{\sigma}\right)$ intervals, $\sigma \in[0,1]$. The optimal $O\left(h^{2}\right)$ convergence rate for linears was observed for $\sigma=1$, in a similar vein to the theoretical result in [1. However, taking $\sigma=.75$ as $h \rightarrow 0$ produced order $h^{1.5}$ convergence, as predicted by (3). It was also observed that when the characteristic direction was altered slightly, taking it out of alignment with the mesh, the optimal rate was restored. In view of the results in [4] and [6], it is natural to ask whether an optimal order estimate might be derivable for fairly general nonaligned meshes.

In this paper, we show that the additional assumption of a nonaligned mesh is not sufficient to guarantee an $O\left(h^{n+1}\right)$ convergence rate, even for smooth solutions $u$. For a simple example problem essentially the same as Peterson's, but with a nonaligned mesh, we show theoretically (for $n=0$ ) and computationally (for $n=0,1,2,3)$ that an $L^{2}$ convergence rate of order $h^{n+1 / 2}$ is realizable. The key factor determining the order of convergence in our example is the distribution of frequencies present in the error as $h \rightarrow 0$.

The basis of our example is a smoothly, periodically varying mesh which induces corresponding sinusoidal error components in the DG approximation. This enables us to direct the error, to a maximal extent, into lower frequency modes which are approximated, as opposed to damped. Such modes persist as the computation proceeds, creating the possibility of $h^{n+1 / 2}$ convergence. As $n$ increases, the range of mesh frequencies for which our example shows suboptimal order convergence is confined to an increasingly narrow, high frequency band. More specifically, if the wavenumber of the mesh perturbation is $p$, our example gives $h^{n+1 / 2}$ convergence 
for $p$ approaching infinity in proportion to $h^{-\frac{2 n+1}{2 n+2}}$ as $h \rightarrow 0$, and $h^{n+1}$ convergence occurs for $p \leq O\left(h^{-\frac{n}{n+1}}\right)$.

An outline of the paper is as follows. In $\S 2$, we describe our example problem and mesh. Then in $\S 3$, we give a simple closed form representation for the corresponding DG approximation $u_{h}$ and its error. In $\S 4$, we prove that for $n=0$ a convergence rate proportional to $\sqrt{h}$ is achievable, indicating sharpness of (3) for our example. In $\S 5$, we provide computational results for $n \leq 3$ and infer a general formula, valid for $n \geq 0$, for the order of convergence achieved by our example problem. Finally, we provide an analytical interpretation of this formula in $\S 6$.

\section{EXAMPLE PROBLEM}

For our example problem, we use the following simplified version of (1) with $\Omega=(-\infty, \infty) \times(0, \pi)$ :

$$
\begin{cases}\frac{\partial u}{\partial y}=0, & (x, y) \in \Omega, \\ u(x, 0)=x^{n+1}, & x \in(-\infty, \infty),\end{cases}
$$

whose solution is $u(x, y)=x^{n+1}$ for $(x, y) \in \Omega$. The $n=1$ version of (4) on the unit square was the basis of the results in [4.

To define our mesh $\tau_{h}$, we start with a uniform discretization of $(-\infty, \infty)$ :

$$
t_{i}=i h, \quad i=0, \pm 1, \pm 2, \ldots
$$

where $h=\frac{\pi}{N}$. We assume $N=p q$, where $p$ and $q$ are positive integers, and define a periodically perturbed set of grid points $\left\{x_{i}\right\}$ by

$$
x_{i}=\psi\left(t_{i}\right), \quad i=0, \pm 1, \pm 2, \ldots
$$

where

$$
\psi(t)=t+\theta \frac{\sin p t}{p}, \quad \theta \in[0,1) .
$$

A single period of the perturbation extends from $x_{i}$ to $x_{i+2 q}$ and spans a distance $\Delta x=\frac{2 \pi}{p}$. As grid points over $y \in[0, \pi]$, we take

$$
y_{j}=x_{j}, \quad j=0,1, \ldots, N \text {. }
$$

In the strip $(-\infty, \infty) \times\left[y_{j-1}, y_{j}\right]$, we obtain our triangles by connecting all triplets

$$
\left\{\left(x_{i-1}, y_{j-1}\right),\left(x_{i}, y_{j}\right),\left(x_{i+1}, y_{j-1}\right)\right\}, \quad\left\{\left(x_{i}, y_{j}\right),\left(x_{i+1}, y_{j-1}\right),\left(x_{i+2}, y_{j}\right)\right\}
$$

for $i=j, j \pm 2, j \pm 4, \ldots$. An illustration of the resulting mesh $\tau_{h}$ is given in Figure 1 .

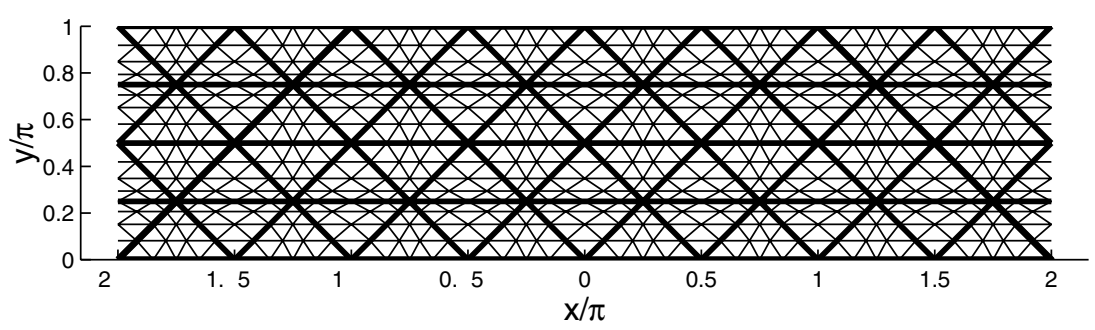

Figure 1. Computational mesh corresponding to $\theta=1 / 3, p=$ $4, q=4$ 
It is easy to verify that $\tau_{h}$ is quasiuniform. For

$$
\begin{aligned}
x_{i+1}-x_{i} & =h+\frac{\theta}{p}\left\{\sin p\left(t_{i}+\frac{h}{2}+\frac{h}{2}\right)-\sin p\left(t_{i}+\frac{h}{2}-\frac{h}{2}\right)\right\} \\
& =h\left[1+\theta \frac{\sin \frac{p h}{2}}{\frac{p h}{2}} \cos p\left(t_{i}+\frac{h}{2}\right)\right] \in[(1-\theta) h,(1+\theta) h] .
\end{aligned}
$$

Thus the triangles in the mesh have width $x_{i+1}-x_{i-1} \in[2(1-\theta) h, 2(1+\theta) h]$ and height $y_{j+1}-y_{j} \in[(1-\theta) h,(1+\theta) h]$. Moreover, along the triangle sides, with unit outer normal $\mathbf{n}$,

$$
|\alpha \cdot \mathbf{n}| \geq \frac{1}{\sqrt{1+\left(\frac{1+\theta}{1-\theta}\right)^{2}}}>0 .
$$

Therefore all triangle sides are uniformly bounded away from the characteristic direction.

In the next section, we will see that the error $e \equiv u-u_{h}$ is $\frac{2 \pi}{p}$-periodic in $x$. Taking norms in a manner that reflects the periodicity of $e$, we define $\|e\|_{\Omega}=$ $\|e\|_{L^{2}([-\pi, \pi] \times[0, \pi))}$ and $\left|e^{-}\right|_{\Gamma_{\text {out }}(\Omega)}=\left|e^{-}\right|_{L^{2}([-\pi, \pi] \times\{y=\pi\})}$. In general, for a $2 \pi-$ periodic function, we shall denote by $|\cdot|$ and $|\cdot|_{m}$ the corresponding $L^{2}$ norm and $H^{m}$ seminorm over $[-\pi, \pi]$.

Our goal will be to show that $p$ and $q$ can be chosen to make $\left|e^{-}\right|_{\Gamma_{\text {out }}(\Omega)} \sim h^{n+1 / 2}$, $\|e\|_{\Omega} \sim h^{n+1 / 2}$, establishing the sharpness of (3) for nonaligned meshes. Here and in what follows, we use the symbol $\sim$ to indicate quantities bounded both from above and below. For example, $\left|e^{-}\right|_{\Gamma_{\text {out }}(\Omega)} \sim h^{n+1 / 2}$ signifies the existence of positive constants $c, C$ such that $c h^{n+1 / 2} \leq\left|e^{-}\right|_{\Gamma_{\text {out }}(\Omega)} \leq C h^{n+1 / 2}$.

Although the domain of (4) is unbounded, the conclusions of this paper also apply to bounded domains. This can be seen, for example, by restricting $\Omega$ to $\tilde{\Omega}=\{(x, y)|| x \mid+y<2 \pi, y \in(0, \pi)\}$, a trapezoid with 45-degree base angles and parallel sides of length $4 \pi, 2 \pi$, and taking as mesh points $\left\{\left(x_{i}, y_{j}\right)|| i \mid+j \leq N\right\}$. From domain of dependence considerations, it follows that the corresponding DG solution $\tilde{u}_{h}$ is the restriction to $\tilde{\Omega}$ of the DG solution to (4).

\section{Closed Form solution}

We begin our analysis by considering a minor generalization of (44):

$$
\begin{cases}\frac{\partial u}{\partial y}=0, & (x, y) \in \Omega, \\ u(x, 0)=u_{0}(x), & x \in(-\infty, \infty),\end{cases}
$$

with solution $u(x, y)=u_{0}(x)$. In each strip $(-\infty, \infty) \times\left[y_{j-1}, y_{j}\right]$, the DG approximation $u_{h}$ for (6) evolves, in parallel, through alternate layers of one-inflow-side (type $I$ ) and two-inflow-side (type $I I$ ) triangles. We denote by $u_{h, j}^{-}$the restriction of $u_{h}^{-}$to the mesh line $y=y_{j}$, and similarly for the error $e_{j}^{-}=u\left(\cdot, y_{j}\right)-u_{h, j}^{-}=$ $u_{0}-u_{h, j}^{-}$. Note that $u_{h, j}^{-}$is a piecewise polynomial of degree $\leq n$ over the subintervals of $y=y_{j}$ (i.e., $\left[x_{i}, x_{i+2}\right], i=j, j \pm 2, j \pm 4, \ldots$ ). We denote by $P_{j}$ the corresponding $L^{2}$ projection into this subspace, and define

$$
\epsilon_{j}=\left(I-P_{j}\right) u_{0} .
$$


Lemma 3.1. The DG approximation for (6) satisfies, for $k=0,1, \ldots, N$,

$$
\begin{aligned}
& u_{h, k}^{-}=P_{k} \cdots P_{0} u_{0}, \\
& e_{k}^{-}=\sum_{j=0}^{k} P_{k} \cdots P_{j+1} \epsilon_{j},
\end{aligned}
$$

where empty operator products (e.g., in (8) for $j=k$ ) are interpreted as the identity. In addition, if $u_{0}=x^{n+1}$ or $u_{0}$ is a $\frac{2 \pi}{p}$-periodic function, then $e=u-u_{h}$ is also $\frac{2 \pi}{p}$-periodic and

$$
\max _{k=0,1, \ldots, N}\left|e_{k}^{-}\right| \leq C h^{n+1 / 2}\left|u_{0}\right|_{n+1},
$$

equivalently, for $k=0,1, \ldots, N$,

$$
P_{k} \cdots P_{0} u_{0}=u_{0}+\delta, \quad|\delta| \leq C h^{n+1 / 2}\left|u_{0}\right|_{n+1} .
$$

Proof. For a generic triangle $T$, let $u_{h, \text { in }}^{-}(x)=\left.u_{h}^{-}(x, y)\right|_{\Gamma_{\text {in }}(T)}$ and $u_{h, \text { out }}^{-}(x)=$ $\left.u_{h}^{-}(x, y)\right|_{\Gamma_{\text {out }}(T)}$. It is easy to see that for a type I triangle $T$,

$$
u_{h}(x, y)=u_{h, \text { in }}^{-}(x), \quad(x, y) \in T .
$$

Thus $u_{h, \text { out }}^{-}(x)=u_{h, \text { in }}^{-}(x)$ for type I triangles. We can develop a corresponding result for type II trangles $T$ by taking $v_{h}$ in (2) to be an arbitrary polynomial $p_{n}(x)$ of degree $\leq n$, which gives

$$
\iint_{T}\left(u_{h}\right)_{y}(x, y) p_{n}(x) d x d y-\int_{\Gamma_{\text {in }}(T)}\left[u_{h}^{+}(x, y)-u_{h}^{-}(x, y)\right] p_{n}(x) d x=0 .
$$

Assuming $x$ ranges from $x_{i}$ to $x_{i+2}$ on $T$, we have

$$
\int_{x_{i}}^{x_{i+2}}\left[u_{h, \text { out }}^{-}(x)-u_{h, \text { in }}^{-}(x)\right] p_{n}(x) d x=0 .
$$

Note that for a type II triangle, $u_{h, \text { in }}^{-}$is a piecewise polynomial while $u_{h, \text { out }}^{-}$is a single polynomial. Thus for type II triangles,

$$
u_{h, \text { out }}^{-}(x)=P_{\text {out }} u_{h, \text { in }}^{-}(x),
$$

where $P_{\text {out }}$ denotes $L^{2}$ projection into polynomials of degree $\leq n$ over $x \in\left[x_{i}, x_{i+2}\right]$. Applying these relations over strips, $(-\infty, \infty) \times\left[y_{j-1}, y_{j}\right]$, of triangles gives

$$
\begin{aligned}
& u_{h, j}^{-}=P_{j} u_{h, j-1}^{-}, \quad j=1, \ldots, N, \\
& u_{h, 0}^{-}=P_{0} u_{0},
\end{aligned}
$$

whose solution is (7).

Recasting (12) in terms of the error $e=u-u_{h}$, we obtain

$$
e_{j}^{-}=P_{j} e_{j-1}^{-}+\epsilon_{j}
$$

whose solution is (8).

If $u_{0}=x^{n+1}$, then $\epsilon_{j}$ is a scalar multiple, dependent on subinterval size, of the $n+1$ st degree Legendre polynomial mapped to the subintervals of $y=y_{j}$. Therefore, $\epsilon_{j}$ will have period $\frac{2 \pi}{p}$, like the mesh oscillation, as will all subsequent 


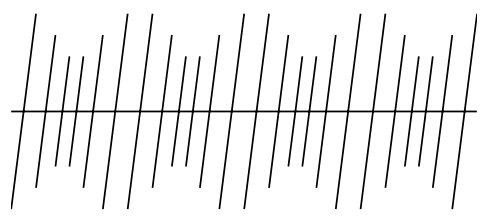

Figure 2. Illustration of $\epsilon_{j}=\left(I-P_{j}\right) x$ for $n=0(\theta=1 / 3, p=$ $4, q=6)$.

projections of $\epsilon_{j}$ appearing in (14) and (8). The same will be true if $u_{0}$ is a $\frac{2 \pi}{p}$ periodic function. In either case, we may therefore regard $\epsilon_{j}$ and $e_{j}^{-}$as periodic $L^{2}[-\pi, \pi]$ functions. Taking norms accordingly, we obtain from (14):

$$
\left|e_{j}^{-}\right|^{2}=\left|P_{j} e_{j-1}^{-}\right|^{2}+\left|\epsilon_{j}\right|^{2} \leq\left|e_{j-1}^{-}\right|^{2}+\left|\epsilon_{j}\right|^{2} \text {. }
$$

Thus

$$
\left|e_{k}^{-}\right|^{2} \leq \sum_{j=0}^{k}\left|\epsilon_{j}\right|^{2} \leq C h^{2 n+1}\left|u_{0}\right|_{n+1}^{2},
$$

where we have used the standard approximation theory estimate $\left|\left(I-P_{j}\right) u_{0}\right| \leq$ $C h^{n+1}\left|u_{0}\right|_{n+1}$. This proves (9), and its alternate form (10).

We may think of $\epsilon_{j}=\left(I-P_{j}\right) u_{0}$ as the $y=y_{j}$ contribution to the error in $u_{h}$; its effect at level $k>j$ is given by $P_{k} \cdots P_{j+1} \epsilon_{j}$. If these subsequent projections of $\epsilon_{j}$ always damp their arguments by a factor $\lambda \leq c<1$, then (8) represents a geometric sum and optimal $O\left(h^{n+1}\right)$ convergence will occur, as in [6].

An illustration of $\epsilon_{j}$ is given in Figure 2 for our example problem (4) with $n=0$. Note that $\epsilon_{j}$ is dominated by high frequency (wavelength $\sim h$ ) components - these will be damped as $u_{h}$ evolves through subsequent levels. However, $\epsilon_{j}$ is a $\frac{2 \pi}{p}$-periodic function, a result of the mesh oscillation; therefore, it also contains lower frequency components of wavenumber $\sim p$. For an appropriately related $p$ and $h$ (equivalently, $p$ and $q$ since $h=\frac{\pi}{p q}$ ) these modes will be approximated, as opposed to damped, by subsequent projections, resulting in a suboptimal order of convergence. In the next section, we will show how to produce $\sqrt{h}$ convergence for $n=0$ via this effect, thus demonstrating sharpness of (3) for our example problem with piecewise constant approximation.

\section{AnALYSis FOR $n=0$}

We now restrict our attention to the case of piecewise constant approximation of (4) with $n=0$. A straightforward calculation shows that $\epsilon_{j}$ contains a lower frequency component with wavenumber $\sim p$ and amplitude $\sim p h^{2}$. However, it is more illuminating to examine $\epsilon_{j}$ after it has undergone one subsequent projection, whereupon this lower frequency component has been "extracted" from $\epsilon_{j}$. That is the content of the following lemma.

Lemma 4.1. For the example problem (4) with $n=0$,

$$
P_{j+1} \epsilon_{j}=\theta p h^{2}\left(\sin p t+\delta_{1}\right), \quad\left|\delta_{1}\right| \leq C p h \quad\left(\leq C q^{-1}\right) .
$$

Proof. Let $\left[x_{i-1}, x_{i+1}\right]$ be a subinterval of mesh line $y=y_{j+1}$. The value of $P_{j+1} \epsilon_{j}$ on $\left[x_{i-1}, x_{i+1}\right]$ is determined by $\epsilon_{j}$ on interlaced subintervals $\left[x_{i-2}, x_{i}\right],\left[x_{i}, x_{i+2}\right]$ of 
$y=y_{j}$. We have $\left.\epsilon_{j}\right|_{\left[x_{i}, x_{i+2}\right]}=x-\frac{1}{2}\left(x_{i}+x_{i+2}\right)=\left(x-x_{i+1}\right)-\frac{1}{2}\left(x_{i}-2 x_{i+1}+x_{i+2}\right)$ and analogously for $\left.\epsilon_{j}\right|_{\left[x_{i-2}, x_{i}\right]}$. Thus for $x \in\left[x_{i-1}, x_{i+1}\right]$,

$$
\begin{aligned}
P_{j+1} \epsilon_{j}= & \frac{1}{x_{i+1}-x_{i-1}} \int_{x_{i-1}}^{x_{i+1}} \epsilon_{j}(x) d x \\
= & \frac{1}{x_{i+1}-x_{i-1}}\left\{\frac{\left(x_{i}-x_{i-1}\right)^{2}}{2}-\left(x_{i}-x_{i-1}\right)\left(\frac{x_{i-2}-2 x_{i-1}+x_{i}}{2}\right)\right. \\
& \left.-\frac{\left(x_{i+1}-x_{i}\right)^{2}}{2}-\left(x_{i+1}-x_{i}\right)\left(\frac{x_{i}-2 x_{i+1}+x_{i+2}}{2}\right)\right\} \\
& =-\left(\frac{x_{i-1}-2 x_{i}+x_{i+1}}{2}-\frac{x_{i}-x_{i-1}}{x_{i+1}-x_{i-1}}\left(\frac{x_{i-2}-2 x_{i-1}+x_{i}}{2}\right)\right. \\
& =-\frac{x_{i+1}-x_{i-1}}{2}\left[\frac{x_{i}-2 x_{i+1}+x_{i+2}}{2}\right) \\
= & -h^{2} \psi^{\prime \prime}\left(\tau_{i}\right), \quad \tau_{i} \in\left(\min \left\{\tau_{i, 1}, \tau_{i, 2}, \tau_{i, 3}\right\}, \max \left\{\tau_{i, 1}, \tau_{i, 2}, \tau_{i, 3}\right\}\right) \subset\left(t_{i-2}, t_{i+2}\right) \\
= & \theta p h^{2}\left(\sin p t+x_{i}(t)\right),
\end{aligned}
$$

where $t$ is the inverse image of $x$ under $\psi$, and $\delta_{1}(t)=\sin p \tau_{i}-\sin p t$. Extending the definition of $\delta_{1}(t)$ to $t \in(-\infty, \infty)$ in the obvious way, we obtain (15).

Before proceeding further, we note that $q=1$ (one subinterval per mesh period) is a degenerate case for this lemma. It gives $\psi(t)=t$ for (5). Thus $\psi^{\prime \prime}=0$ in the preceding proof, implying $P_{j+1} \epsilon_{j}=0, e_{k}^{-}=\epsilon_{k}$ in (8), hence optimal order convergence.

We next show that for $p \leq O\left(h^{-1 / 2}\right)$ as $h \rightarrow 0, P_{j+1} \epsilon_{j}$ will be approximated, and thus persist, when subsequent projections $P_{j+2}, \ldots, P_{N}$ are applied, leading to a suboptimal convergence rate.

Theorem 4.1. For the example problem (4) with $n=0$, let $p=\mu h^{-\sigma}$ where $\sigma \in\left[0, \frac{1}{2}\right]$ and $\mu>0$ (with $\mu$ sufficiently small if $\sigma=\frac{1}{2}$ ). Then as $h \rightarrow 0$, both $\left|u-u_{h}^{-}\right|_{\Gamma_{\text {out }}(\Omega)}$ and $\left\|u-u_{h}\right\|_{\Omega}$ approach zero in proportion to $h^{1-\sigma}$. In particular, the boundary and interior estimates given in (3) are attained for $\sigma=\frac{1}{2}$.

Proof. The effect of $\epsilon_{j}$ at a subsequent level $k>j$ is given by

$$
P_{k} \cdots P_{j+1} \epsilon_{j}=\theta p h^{2}\left(P_{k} \cdots P_{j+2} \sin p t+\delta_{2}\right), \quad\left|\delta_{2}\right| \leq C p h .
$$

Applying (10) over $\Omega \cap\left(y_{j+2}, \pi\right)$ with initial condition $u_{0}=\sin p t$ on $y=y_{j+2}$, and using the fact that $|\sin p t|_{1} \leq C p$ (since $H^{1}$-norms with respect to $x$ and $t$ are compatible), we obtain for $k>j$,

$$
P_{k} \cdots P_{j+1} \epsilon_{j}=\theta p h^{2}\left(\sin p t+\delta_{3}\right), \quad\left|\delta_{3}\right| \leq C p \sqrt{h} .
$$

It follows from (8) that

$$
e_{k}^{-}=\theta p h^{2} k\left(\sin p t+\delta_{4}\right)+\epsilon_{k}, \quad\left|\delta_{4}\right| \leq C p \sqrt{h} .
$$


Now suppose $p=\mu h^{-\sigma}$ as $h \rightarrow 0$ where $\sigma \geq 0$ and $\mu>0$ are constants. Then (16) becomes

$$
e_{k}^{-}=\theta \mu k h^{2-\sigma}\left(\sin p t+\delta_{4}\right)+\epsilon_{k}, \quad\left|\delta_{4}\right| \leq C \mu h^{\frac{1}{2}-\sigma} .
$$

For $\sigma \in\left[0, \frac{1}{2}\right),\left|\delta_{4}\right| \rightarrow 0$ as $h \rightarrow 0$, and for $\sigma=\frac{1}{2}$, we may choose $\mu$ small enough to ensure that $\left|\delta_{4}\right|<|\sin p t|$. In addition, $\left|\epsilon_{k}\right|=\left|\left(I-P_{k}\right) x\right| \leq C h$. We conclude, for $\sigma \in\left[0, \frac{1}{2}\right]$,

$$
\left|e_{k}^{-}\right| \sim k h^{2-\sigma} \quad \text { as } h \rightarrow 0 .
$$

Taking $k=N\left(=\frac{\pi}{h}\right)$, we get $\left|e_{N}^{-}\right|=\left|u-u_{h}^{-}\right|_{\Gamma_{\text {out }}(\Omega)} \sim h^{1-\sigma}$.

Next we obtain an interior estimate for $e=u-u_{h}$, a piecewise linear for our example with $n=0$. For a type I triangle $T$, it follows from (11) and scaling that $c h\left|e^{-}\right|_{\Gamma_{\text {in }}(T)}^{2} \leq\|e\|_{T}^{2} \leq C h\left|e^{-}\right|_{\Gamma_{\text {in }}(T)}^{2}(c, C$ positive constants), while for a type II triangle $T$, it is easy to show (see for example [6]) that $\|e\|_{T}^{2} \leq C h\left|e^{-}\right|_{\Gamma_{\text {in }}(T)}^{2}$. Let $W_{j}=\Omega \cap\left[y_{j}, y_{j+1}\right]$ and $W_{j, 1}, W_{j, 2}$ be the union of the type I and type II triangles of $W_{j}$. Then $\|e\|_{W_{j, 2}}^{2} \leq C h\left|e^{-}\right|_{\Gamma_{\text {out }}\left(W_{j, 1}\right)}^{2}=C h\left|e_{j}^{-}\right|^{2}$ and $c h\left|e_{j}^{-}\right|^{2} \leq\|e\|_{W_{j, 1}}^{2} \leq C h\left|e_{j}^{-}\right|^{2}$. Therefore, $\operatorname{ch}\left|e_{j}^{-}\right|^{2} \leq\|e\|_{W_{j}}^{2} \leq C h\left|e_{j}^{-}\right|^{2}$, and

$$
\|e\|_{\Omega}^{2} \sim h \sum_{j=0}^{N-1}\left|e_{j}^{-}\right|^{2} .
$$

This desired interior estimate, $\|e\|_{\Omega} \sim h^{1-\sigma}$, follows from (18) and (19).

As yet, we have said nothing about the convergence rate of $u_{h}$ for our example problem in the case where $p=\mu h^{-\sigma}, \sigma \in\left(\frac{1}{2}, 1\right]$. We now address this. Let $S=\left\{v \in L^{2}[-\pi, \pi] \mid v\right.$ is $\frac{2 \pi}{p}$ - periodic, $\left.(v, 1)=0\right\}$, and let $S_{h, j}$ be the intersection of $S$ with the space of piecewise constants over the subintervals along $y=y_{j}$. In what follows, we assume $q \geq 2$. (For the degenerate case $q=1$, previously noted following the proof of Lemma 4.1, there is only one subinterval per mesh period, and $S_{h, j}=\{0\}$.)

Referring to (8), we observe that the error components $P_{k} \cdots P_{j+1} \epsilon_{j}$ are in $S_{h, k}$. More specifically, $P_{j+1}$ maps $\epsilon_{j}$ into $S_{h, j+1}$, while for $l \geq j+2, P_{l}$ maps an operand in $S_{h, l-1}$ into $S_{h, l}$. Focusing on the latter effect, we define $\left|P_{l}\right|=$ $\max _{w \in S_{h, l-1},|w|=1}\left|P_{l} w\right|$. Here $\left|P_{l} w\right|$ measures the extent to which $w \in S_{h, l-1}$ can be approximated in $S_{h, l}$. Since $S_{h, l-1} \cap S_{h, l}=\{0\},\left|P_{l}\right|<1$ and the error components in (8) are damped as the computation progresses. We quantify this damping as follows.

Lemma 4.2. As a map from $S_{h, j-1}$ to $S_{h, j}$, $P_{j}$ satisfies

$$
\left|P_{j}\right| \leq 1-\gamma(p h)^{2},
$$

where $\gamma$ is a positive constant, depending only on the mesh parameter $\theta$.

Proof. Without loss of generality, we will show this for $P_{1}$, as a map from $S_{h, 0}$ to $S_{h, 1}$. The mesh points of $S_{h, 0}$ are $\cdots<x_{-2}<x_{0}<x_{2}<\cdots$ while those of $S_{h, 1}$ are $\cdots<x_{-1}<x_{1}<x_{3}<\cdots$. For arbitrary nonzero $v_{h, 0} \in S_{h, 0}$ and $v_{h, 1} \in S_{h, 1}$, let

$$
v_{i}= \begin{cases}v_{h, 0}\left(x_{i}\right), & i \text { odd }, \\ v_{h, 1}\left(x_{i}\right), & i \text { even }\end{cases}
$$


and define a continuous piecewise linear $v_{h}$ over the combined mesh $\cdots x_{-1}<x_{0}<$ $x_{1}<x_{2}<\cdots$ by $v_{h}\left(x_{i}\right)=v_{i}$ for all $i$. From

$$
\int_{x_{i}}^{x_{i+1}} v_{h} d x=\frac{x_{i+1}-x_{i}}{2}\left(v_{i}+v_{i+1}\right)=\frac{1}{2}\left\{\int_{x_{i}}^{x_{i+1}} v_{h, 0}+\int_{x_{i}}^{x_{i+1}} v_{h, 1} d x\right\},
$$

it follows that $\left(v_{h}, 1\right)=\frac{1}{2}\left\{\left(v_{h, 0}, 1\right)+\left(v_{h, 1}, 1\right)\right\}$, implying $v_{h} \in S$. In addition,

$$
\int_{x_{i}}^{x_{i+1}} v_{h}^{2} d x=\frac{x_{i+1}-x_{i}}{3}\left(v_{i}^{2}+v_{i} v_{i+1}+v_{i+1}^{2}\right),
$$

and for $x \in\left(x_{i}, x_{i+1}\right), v_{h, 0}(x)=v_{i}$ or $v_{i+1}$. We assume the former, without loss of generality for our purposes, and apply the arithmetic-geometric mean inequality in the form $v_{i} v_{i+1} \geq-\left(\frac{1}{4} v_{i}^{2}+v_{i+1}^{2}\right)$ to obtain

$$
\int_{x_{i}}^{x_{i+1}} v_{h}^{2} d x \geq \frac{x_{i+1}-x_{i}}{4} v_{i}^{2}=\frac{1}{4} \int_{x_{i}}^{x_{i+1}} v_{h, 0}^{2} d x .
$$

Thus $\left|v_{h, 0}\right| \leq 2\left|v_{h}\right|$.

Defining $h_{\min }=\min _{i}\left\{x_{i+1}-x_{i}\right\}$, we have

$$
\begin{aligned}
\int_{x_{i}}^{x_{i+1}}\left(v_{h, 1}-v_{h, 0}\right)^{2} d x & =\left(x_{i+1}-x_{i}\right)\left(v_{i+1}-v_{i}\right)^{2} \\
& =\left(x_{i+1}-x_{i}\right)^{3}\left(\frac{v_{i+1}-v_{i}}{x_{i+1}-x_{i}}\right)^{2} \\
& \geq h_{\min }^{2} \int_{x_{i}}^{x_{i+1}}\left(v_{h}^{\prime}\right)^{2} d x
\end{aligned}
$$

Hence $\left|v_{h, 1}-v_{h, 0}\right| \geq h_{\min }\left|v_{h}\right|_{1}$, and

$$
\begin{aligned}
\frac{\left|v_{h, 1}-v_{h, 0}\right|}{\left|v_{h, 0}\right|} & \geq h_{\min } \frac{\left|v_{h}\right|_{1}}{\left|v_{h, 0}\right|} \\
& \geq \frac{h_{\min }}{2} \frac{\left|v_{h}\right|_{1}}{\left|v_{h}\right|} \\
& \geq \frac{h_{\min }}{2} \min _{v \in S \cap H^{1}[-\pi, \pi], v \neq 0} \frac{|v|_{1}}{|v|} \\
& =\frac{p h_{\min }}{2},
\end{aligned}
$$

where (via a Fourier series expansion) the minimum over $S \cap H^{1}[-\pi, \pi]$ occurs for $v$ a nonzero linear combination of $\cos p x, \sin p x$; equivalently, the complex functions $e^{ \pm i p x}$. Note that the above separation result holds for any set of $\frac{2 \pi}{p}$-periodic grid points $x_{i}$, not just our particular ones.

Taking $v_{h, 1}=P_{1} v_{h, 0}$, we obtain

$$
\frac{\left|\left(I-P_{1}\right) v_{h, 0}\right|}{\left|v_{h, 0}\right|} \geq \frac{p h_{\min }}{2} .
$$


Thus

$$
\begin{aligned}
\frac{\left|P_{1} v_{h, 0}\right|}{\left|v_{h, 0}\right|} & =\sqrt{\frac{\left|v_{h, 0}\right|^{2}-\left|\left(I-P_{1}\right) v_{h, 0}\right|^{2}}{\left|v_{h, 0}\right|^{2}}} \\
& \leq \sqrt{1-\frac{\left(p h_{\min }\right)^{2}}{4}} \\
& \leq 1-\frac{p^{2} h_{\min }^{2}}{8} .
\end{aligned}
$$

(Note: $1-\frac{p^{2} h_{\min }^{2}}{8} \geq 1-\frac{p^{2} h^{2}}{8}=1-\frac{\pi^{2}}{8 q^{2}}>0$.) The desired bound now follows from the quasi-uniformity of the mesh.

We show next how (20) leads to an estimate of the convergence rate for our example problem for the full range of possible $\sigma$ values.

Theorem 4.2. For the example problem (4) with $n=0$, let $p=\mu h^{-\sigma}$ where $\sigma \in[0,1]$ and $\mu>0$ (with $\mu$ sufficiently small if $\sigma=1$ ). Then

$$
\left|u-u_{h}^{-}\right|_{\Gamma_{\text {out }}(\Omega)}+\left\|u-u_{h}\right\|_{\Omega} \leq C h^{\nu}, \quad \nu=\frac{1}{2}+\left|\sigma-\frac{1}{2}\right| .
$$

Proof. We express our bound (20) in the form

$$
\left|P_{j}\right| \leq \lambda, \quad \lambda=1-\gamma(p h)^{2}=e^{-\gamma(p h)^{2}+O(p h)^{4}} .
$$

Applying (8), (15), and (22), we infer that the error for our example problem satisfies

$$
\begin{aligned}
\left|e_{k}^{-}\right| & \leq\left|\epsilon_{k}\right|+\sum_{j=0}^{k-1} \lambda^{k-1-j}\left|P_{j+1} \epsilon_{j}\right| \\
& \leq C h+\left(\sum_{j=0}^{k-1} \lambda^{j}\right)\left(C p h^{2}\right) \\
& =C h\left\{1+\frac{1-\lambda^{k}}{\gamma p h}\right\} \\
& \leq C h\left\{1+\frac{1-\lambda^{N}}{\gamma p h}\right\} .
\end{aligned}
$$

From (22) and the fact that $N=\frac{\pi}{h}$, we have $\lambda^{N}=e^{-\gamma \pi p^{2} h\left(1+O(p h)^{2}\right)}$. For $p=\mu h^{-\sigma}$, this becomes

$$
\lambda^{N}=e^{-\gamma \pi \mu^{2} h^{1-2 \sigma}\left(1+\mu^{2} O\left(h^{2-2 \sigma}\right)\right)} .
$$

Thus as $h \rightarrow 0$, we have

$$
\begin{aligned}
\sigma \in\left[0, \frac{1}{2}\right): & \lambda^{N}=1-\gamma \pi \mu^{2} h^{1-2 \sigma}+\mu^{4} O\left(h^{1-2 \sigma}\right)^{2}, \\
\sigma=\frac{1}{2}: & \lambda^{N} \rightarrow e^{-\gamma \pi \mu^{2}}, \\
\sigma \in\left(\frac{1}{2}, 1\right]: & \lambda^{N} \rightarrow 0,
\end{aligned}
$$

where $\mu$ is assumed to be sufficiently small if $\sigma=1$. We conclude from (23) and (25) that for $k \leq N$, 


$$
\left|e_{k}^{-}\right| \leq \begin{cases}C h^{1-\sigma}, & \sigma \in\left[0, \frac{1}{2}\right], \\ C h^{\sigma}, & \sigma \in\left(\frac{1}{2}, 1\right] .\end{cases}
$$

These bounds also apply to $\left\|u-u_{h}\right\|_{\Omega}$ via (19).

For $\sigma \in\left[0, \frac{1}{2}\right]$, this estimate is consistent with that of Theorem 4.1; therefore, it is sharp for $\sigma$ in this range. In addition, our computational results (next section) suggest that it is also sharp for $\sigma \in\left(\frac{1}{2}, 1\right]$.

It is instructive to consider the DG approximation for (6) with the given mesh, $p=\mu h^{-\sigma}$, and ( $h$-dependent) initial condition $u(x, 0)=e^{i p x}$. As $h \rightarrow 0$, we have for the corresponding DG approximation $u_{h}$ :

$$
\left.u_{h}^{-}\right|_{\Gamma_{\text {out }}(\Omega)} \rightarrow \begin{cases}e^{i p x}, & \sigma \in\left[0, \frac{1}{2}\right), \\ 0, & \sigma \in\left(\frac{1}{2}, 1\right] .\end{cases}
$$

The first part of (26) is a straightforward consequence of the basic estimate (3) or (10). The second part (since $P_{0} e^{i p x} \in S_{h, 0}$ ) is implied by $\left|u_{h, N}^{-}\right|=\left|P_{N} \cdots P_{0} e^{i p x}\right| \leq$ $\lambda^{N}\left|e^{i p x}\right|$ and (25). Thus $\sigma=\frac{1}{2}$ lies at the threshold between approximation and exponential damping of $e^{i p x}$. Returning to our example problem (4) with $u(x, 0)=$ $x$ and referring again to (15), we see that the choice $\sigma=\frac{1}{2}$ maximizes the size of the lower frequency portion of $\epsilon_{j}$ while retaining its approximability as $h \rightarrow 0$.

\section{Computational Results}

In this section, we present numerical results for our example problem (44), discretized over the mesh defined in $\S 2$. Table 1 shows computed outflow errors $\left|u-u_{h}^{-}\right|_{\Gamma_{\text {out }}(\Omega)}$ for $n=0$ for various values of $\sigma$, plus order of convergence estimates based on consecutive values of $\left|u-u_{h}^{-}\right|_{\Gamma_{\text {out }}(\Omega)}$. Values chosen for $N\left(=\frac{\pi}{h}\right)$ are such that $p$ and $q$ are integers. The value of the mesh parameter $\theta$ was fixed at $\frac{1}{3}$. For $\sigma=\frac{1}{4}$ and $\sigma=\frac{1}{2}$, the computed orders of convergence approach the theoretical limits $h^{\frac{3}{4}}, h^{1 / 2}$ given by (16), while for $\sigma=\frac{3}{4}$ and $\sigma=1$, the observed $h^{3 / 4}$ and $h^{1}$ rates are consistent with (21). The estimate (21) thus gives the correct order of convergence for all $\sigma$ used in our computations with $n=0$.

Tables 2 and 3 give analogous computational results for $n=1$ and $n=2$. For $n=1$, optimal $O\left(h^{2}\right)$ convergence occurs for $\sigma \in\left[0, \frac{1}{2}\right]$, while the $O\left(h^{1.5}\right)$ bound (31) is achieved for $\sigma=\frac{3}{4}$, as in [4]. For $n=2$, we get optimal order convergence for $\sigma \in\left[0, \frac{2}{3}\right]$, and $O\left(h^{2.5}\right)$ (as in (3) ) for $\sigma=\frac{5}{6}$.

A single formula which describes our observed orders of convergence is:

$$
\left|u-u_{h}^{-}\right|_{\Gamma_{\text {out }}(\Omega)} \sim \begin{cases}h^{n+1}, & \sigma \in\left[0, \frac{n}{n+1}\right], \\ h^{(2 n+1)-\sigma(n+1)}, & \sigma \in\left(\frac{n}{n+1}, \frac{2 n+1}{2 n+2}\right], \\ h^{(n+1) \sigma}, & \sigma \in\left(\frac{2 n+1}{2 n+2}, 1\right] .\end{cases}
$$

As further validation of (27), we performed experiments with (i) $n=1, \sigma=\frac{2}{3}$, (ii) $n=1, \sigma=\frac{5}{6}$, (iii) $n=2, \sigma=\frac{3}{4}$, (iv) $n=3, \sigma=\frac{3}{4}$, (v) $n=3, \sigma=\frac{5}{6}$,

(vi) $n=3, \sigma=\frac{7}{8}$, and observed results consistent with the predicted orders $\left(1 \frac{2}{3}, 1 \frac{2}{3}, 2 \frac{3}{4}, 4,3 \frac{2}{3}, 3 \frac{1}{2}\right)$. A plot of the orders in (27) vs. $\sigma$ for $n=0,1,2,3$ is shown in Figure 3. As $n$ increases, suboptimal order convergence occurs for an increasingly narrow band of high frequency mesh perturbations centered about $\sigma=\frac{2 n+1}{2 n+2}$, which gives $h^{n+1 / 2}$ convergence. 
TABLE 1. Results for $n=0 ; p=(N / 4)^{\sigma} \sim h^{-\sigma}$.

\begin{tabular}{||r||l|l|l|l|l|l|l|l||}
\hline \multirow{2}{*}{$N$} & \multicolumn{2}{|c|}{$\sigma=1 / 4$} & \multicolumn{2}{c|}{$\sigma=1 / 2$} & \multicolumn{2}{c|}{$\sigma=3 / 4$} & \multicolumn{2}{c||}{$\sigma=1$} \\
\cline { 2 - 8 } & error & order & error & order & error & order & error & order \\
\hline \hline 4 & 1.429 & & 1.429 & & 1.429 & & 1.429 & \\
64 & $1.745(-1)$ & .759 & $2,224(-1)$ & .671 & $1.616(-1)$ & .786 & $9.889(-2)$ & .963 \\
324 & $5.268(-2)$ & .738 & $9.448(-2)$ & .528 & $4.594(-2)$ & .775 & $1.593(-2)$ & 1.000 \\
1,024 & $2.244(-2)$ & .742 & $5.272(-2)$ & .507 & $1.905(-2)$ & .765 & $6.180(-3)$ & 1.000 \\
5,184 & $6.702(-3)$ & .745 & $2,336(-2)$ & .502 & $5.573(-3)$ & .758 & $1.221(-4)$ & 1.000 \\
\hline
\end{tabular}

TABLE 2. Results for $n=1 ; p=(N / 4)^{\sigma} \sim h^{-\sigma}$.

\begin{tabular}{||r||l|l|l|l||l|l|l|l||}
\hline \multirow{2}{*}{$N$} & \multicolumn{2}{|c|}{$\sigma=1 / 4$} & \multicolumn{2}{c|}{$\sigma=1 / 2$} & \multicolumn{2}{c|}{$\sigma=3 / 4$} & \multicolumn{2}{c||}{$\sigma=1$} \\
\cline { 2 - 8 } & error & order & error & order & error & order & error & order \\
\hline \hline 4 & $6.159(-1)$ & & $6.159(-1)$ & & $6.159(-1)$ & & $6.159(-1)$ & \\
64 & $2.287(-3)$ & 2.018 & $2.621(-3)$ & 1.969 & $5.222(-3)$ & 1.720 & $1.066(-2)$ & 1.463 \\
324 & $8.848(-5)$ & 2.005 & $1.029(-4)$ & 1.996 & $4.354(-4)$ & 1.532 & $6.588(-4)$ & 1.717 \\
1,024 & $8.844(-6)$ & 2.001 & $1.032(-5)$ & 1.999 & $7.719(-5)$ & 1.503 & $6.645(-5)$ & 1.993 \\
5,184 & $3.449(-7)$ & 2.000 & $4.028(-7)$ & 2.000 & $6.792(-6)$ & 1.499 & $2.593(-6)$ & 2.000 \\
\hline
\end{tabular}

TABLe 3. Results for $n=2 ; p=N^{\sigma} \sim h^{-\sigma}$.

\begin{tabular}{||r||l|l||l|l||l|l||l|l||}
\hline \multirow{2}{*}{$N$} & \multicolumn{2}{|c|}{$\sigma=1 / 2$} & \multicolumn{2}{c|}{$\sigma=2 / 3$} & \multicolumn{2}{c|}{$\sigma=5 / 6$} & \multicolumn{2}{c||}{$\sigma=1$} \\
\cline { 2 - 8 } & error & order & error & order & error & order & error & order \\
\hline \hline 1 & $1.354(1)$ & & $1.354(1)$ & & $1.354(1)$ & & $1.354(1)$ & \\
64 & $7.399(-5)$ & 2.914 & $1.130(-4)$ & 2.812 & $2.446(-4)$ & 2.626 & $4.798(-5)$ & 3.018 \\
729 & $4.963(-8)$ & 3.004 & $9.867(-8)$ & 2.895 & $6.389(-7)$ & 2.445 & $3.247(-8)$ & 3.000 \\
4,096 & $2.795(-10)$ & 3.001 & $5.808(-10)$ & 2.975 & $8.976(-9)$ & 2.471 & $1.830(-10)$ & 3.000 \\
15,625 & $5.035(-12)$ & 3.000 & $1.057(-11)$ & 2.992 & $3.233(-10)$ & 2.483 & $3.297(-12)$ & 3.000 \\
\hline
\end{tabular}

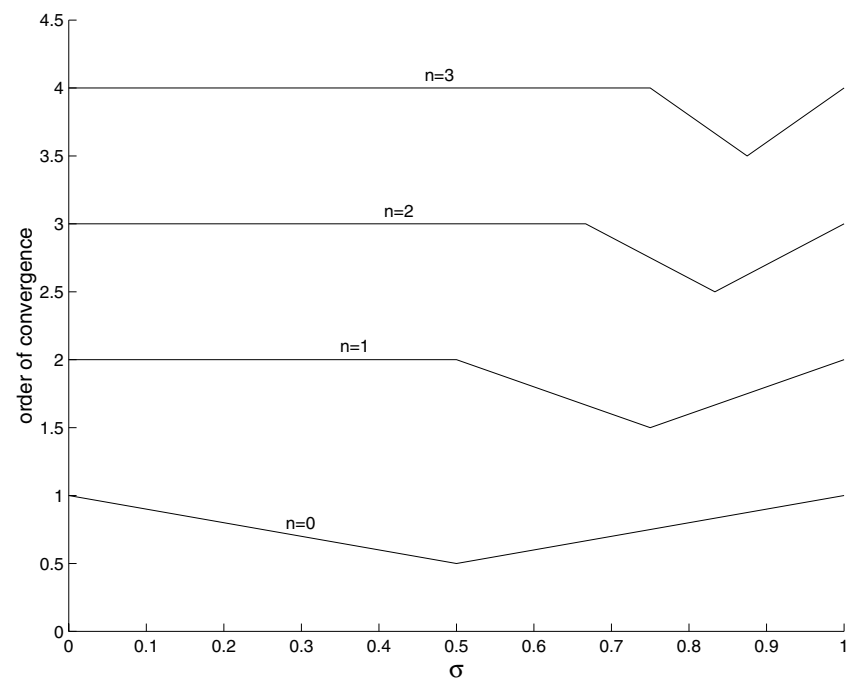

FiguRE 3. Order of convergence vs. $\sigma$ for $n=0,1,2,3$. 


\section{Discussion}

We now provide an analytical interpretation of the order formula (27) for arbitrary $n \geq 0$.

For our example problem (4), the quantity $\epsilon_{j}=\left(I-P_{j}\right) x^{n+1}$ is dominated by high frequency (wavelength $\sim h$ ) components. However, it will also have a lower frequency component $w$ of wavenumber $\sim p$ for which

$$
|w|_{n+1} \leq C p^{n+1}|w|
$$

By estimating the cumulative effect of such error components $w$, we can offer an explanation for (27).

By (10) and (28), the effect of $w$ at a subsequent level $y=y_{k}$ is

$$
P_{k} \cdots P_{j+1} w=w+\nu_{1}, \quad\left|\nu_{1}\right| \leq C h^{n+1 / 2}|w|_{n+1} \leq C h^{n+1 / 2} p^{n+1}|w| .
$$

If, as before, $p=\mu h^{-\sigma}$, then $\left|\nu_{1}\right| \leq C \mu^{n+1} h^{(n+1 / 2)-\sigma(n+1)}$, and we can ensure that $\left|\nu_{1}\right|<|w|$ by taking $\sigma \in\left[0, \frac{2 n+1}{2 n+2}\right]$ (also taking $\mu$ sufficiently small if $\sigma=\frac{2 n+1}{2 n+2}$ ). Thus for $\sigma$ in this range, $w$ will be approximated at subsequent levels, not damped, as $h \rightarrow 0$.

We next estimate the size of $w$. We have

$$
\begin{aligned}
|w|^{2} & =\left|\left(\epsilon_{j}, w\right)\right| \\
& =\left|\left(\left(I-P_{j}\right) x^{n+1},\left(I-P_{j}\right) w\right)\right| \\
& \leq C h^{2 n+2}\left|x^{n+1}\right|_{n+1}|w|_{n+1} .
\end{aligned}
$$

Hence, applying (28),

$$
|w| \leq C p^{n+1} h^{2 n+2} \leq C h^{(2 n+2)-\sigma(n+1)} .
$$

We denote by $E_{w}$ the total contribution of all error components $w$ to the outflow boundary error $\left|u-u_{h}^{-}\right|_{\Gamma_{\text {out }}(\Omega)}$. Since there are $O\left(h^{-1}\right)$ levels in all, therefore $O\left(h^{-1}\right)$ such error components,

$$
E_{w} \leq C h^{(2 n+1)-\sigma(n+1)} \begin{cases}\leq C h^{n+1}, & \sigma \in\left[0, \frac{n}{n+1}\right], \\ =C h^{n+\frac{1}{2}}, & \sigma=\frac{2 n+1}{2 n+2} .\end{cases}
$$

Moreover, if (29) is sharp and the error components $w$ add to, rather than cancel one another, both of which are true for $n=0$, then this estimate for $E_{w}$ is sharp.

To extend the analysis to $\sigma \in\left(\frac{2 n+1}{2 n+2}, 1\right]$, let

$$
S=\left\{v \in L^{2}[-\pi, \pi] \mid v \text { is } \frac{2 \pi}{p}-\text { periodic, }(v, 1)=0\right\}
$$

as before, and $S_{h, j}^{(n)}$ the intersection of $S$ with the space of piecewise $n$th degree polynomials (in general discontinuous) over the subintervals of $y=y_{j}$. Our computational results suggest that $P_{j}$, viewed as a map from $S_{h, j-1}^{(n)}$ to $S_{h, j}^{(n)}$, satisfies a bound of the form $\left|P_{j}\right| \leq \lambda_{n}$ where $\lambda_{n}=1-\gamma_{n}(p h)^{2 n+2}$, a natural extension of Lemma 4.2 to $n \geq 0$. Assuming this to be the case, the error component $w$ generated at $y=y_{j}, j<N$, contributes at most $\left|P_{N} \cdots P_{j+1} w\right| \leq \lambda_{n}^{N-j-1}|w|$ to 
$\left|u-u_{h}^{-}\right|_{\Gamma_{\text {out }}(\Omega)}$. Applying (29) and proceeding as in (23), we obtain

$$
\begin{aligned}
E_{w} & \leq\left(1+\sum_{j=0}^{N-1} \lambda_{n}^{j}\right)\left(C p^{p+1} h^{2 n+2}\right) \\
& \leq C p^{n+1} h^{2 n+2}\left(1+\frac{1-\lambda_{n}^{N}}{\gamma_{n}(p h)^{2 n+2}}\right) .
\end{aligned}
$$

Now for $p=\mu h^{-\sigma}$,

$$
\begin{aligned}
\lambda_{n} & =\exp \left\{-\gamma_{n}(p h)^{2 n+2}\left(1+O(p h)^{2 n+2}\right)\right\} \\
& =\exp \left\{-\gamma_{n}\left(\mu h^{1-\sigma}\right)^{2 n+2}\left(1+O\left(\mu h^{1-\sigma}\right)^{2 n+2}\right\} .\right.
\end{aligned}
$$

Thus

$$
\lambda_{n}^{N}=\exp \left\{-\gamma_{n} \pi \mu^{2 n+2} h^{(2 n+1)-\sigma(2 n+2)}\left[1+\mu^{2 n+2} O\left(h^{(2 n+2)(1-\sigma)}\right)\right]\right\},
$$

and as $h \rightarrow 0$, we have

$$
\begin{aligned}
\sigma \in\left(\frac{2 n+1}{2 n+2}, 1\right]: & \lambda_{n}^{N} \rightarrow 0 \\
\sigma=\frac{2 n+1}{2 n+2}: & \lambda_{n}^{N} \rightarrow \exp \left\{-\gamma_{n} \pi \mu^{2 n+2}\right\} \\
\sigma \in\left[0, \frac{2 n+1}{2 n+2}\right): & \lambda_{n}^{N}=1-\gamma_{n} \pi \mu^{2 n+2} h^{(2 n+1)-\sigma(2 n+2)}(1+\delta),
\end{aligned}
$$

where $\delta \rightarrow 0$ as $h \rightarrow 0$, and $\mu$ is taken sufficiently small if $\sigma=1$. Substituting for $\lambda_{n}^{N}$ in (31), we obtain

$$
E_{w} \leq \begin{cases}C h^{(2 n+1)-\sigma(n+1)}, & \sigma \in\left[0, \frac{2 n+1}{2 n+2}\right], \\ C h^{(n+1) \sigma}, & \sigma \in\left(\frac{2 n+1}{2 n+2}, 1\right] .\end{cases}
$$

This estimate extends (30) to the entire range $\sigma \in[0,1]$. It indicates the presence of a suboptimal order error component in $u-\left.u_{h}^{-}\right|_{\Gamma_{\text {out }}(\Omega)}$ for $\sigma \in\left(\frac{n}{n+1}, 1\right)$, whose maximum $O\left(h^{n+1 / 2}\right)$ amplitude is reached at $\sigma=\frac{2 n+1}{2 n+2}$. This is consistent with our observed formula (27) and describes what we believe to be the mechanism for its occurrence.

Our analysis shows, in essence, how the first several terms of the Fourier expansion of $\epsilon_{j}$ in terms of $\left\{e^{i k p x}, k= \pm 1, \pm 2, \ldots\right\}$ can account for the region of suboptimal order convergence that occurs for our example problem. We have also performed some computations for $\frac{2 \pi}{p}$-periodic grid points $\left\{x_{i}\right\}$ with a jump discontinuity in subinterval size, and observed $h^{n+1 / 2}$ convergence for $\sigma=\frac{2 n+1}{2 n+2}$, as in the case of our smoothly varying mesh, but a larger range of $\sigma$ values giving suboptimal order convergence. Our results for $n=1$ are similar to those reported by Peterson [4. For a discontinuously varying mesh, the Fourier coefficients of the lower frequency portion of $\epsilon_{j}$ can be shown to decay more slowly with $k$, allowing a broader spectrum of Fourier modes to influence the order of convergence.

We state, in conclusion, that a nonaligned mesh is not the key to an improved rate of convergence over the $h^{n+1 / 2}$ estimate in (3). Our analysis and computational results show that an $h^{n+1 / 2}$ rate is achievable for a periodic mesh which generates, to the maximal extent, lower frequency error components which are approximated, not damped, as $h \rightarrow 0$. 


\section{ACKNOWLEDGMENTS}

I thank the referees for their many helpful suggestions for improving this presentation.

\section{REFERENCES}

[1] B. Cockburn, B. Dong, and J. Guzmán, Optimal convergence of the original DG method for the transport-reaction equation on special meshes, Institute for Mathematics and its Applications, University of Minnesota, IMA Preprint Series \# 2147, December, 2006.

[2] C. Johnson and J. Pitkäranta, An analysis of the discontinuous Galerkin method for a scalar hyperbolic equation, Math. Comput. 47 (1986), pp. 285-312.

[3] P. Lesaint and P. A. Raviart On a finite element for solving the neutron transport equation, in Mathematical Aspects of Finite Elements in Partial Differential Equations, C. de Boor, ed., Academic Press, New York, 1974, pp. 89-123. MR0658142 (58:31918)

[4] T. E. Peterson, A note on the convergence of the discontinuous Galerkin method for a scalar hyperbolic equation, SIAM J. Numer. Anal. 28 (1991), pp. 133-140. MR.1083327 (91m:65250)

[5] W. H. Reed and T. R. Hill Triangular mesh methods for the neutron transport equation, Tech. Report LA-UR-73-479, Los Alamos National Laboratory, Los Alamos NM, 1973.

[6] G. R. Richter An optimal-order error estimate for the discontinuous Galerkin method, Math.Comp. 50 (1988), pp. 75-88. MR917819(88j:65197)

Department of Computer Science, Rutgers University, Busch Campus, Piscataway New JeRSEy 08854-8019

E-mail address: richter@cs.rutgers.edu 